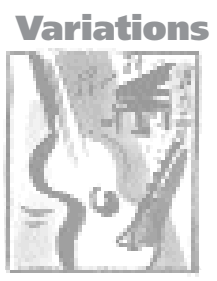

\title{
Italie et Royaume-Uni : les surprises d'une comparaison
}

\author{
par Colin Crouch
}

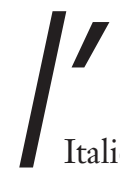

talie et le Royaume-Uni sont des pays très comparables en ce qui concerne la population et le produit intérieur brut et, selon les variations de sa monnaie, chacun des deux peut légèrement dépasser l'autre en termes de revenu par tête. Ils présentent cependant des situations extrêmement contrastées à maints égards. La Grande-Bretagne, bien qu'ayant été la première nation industrielle du monde, n'en a pas moins longtemps gardé dans sa culture de multiples formes d'hostilité à l'industrialisme (Wiener 1981). Le secteur financier, la City de Londres, a d'ailleurs toujours dominé la politique économique, la production industrielle étant moins prioritaire aux yeux des pouvoirs publics. La suprématie de la finance a facilité les rachats et les fusions, façonnant une économie surtout constituée de grandes sociétés. Les petites entreprises industrielles y sont au surplus handicapées par l'absence de tout système réglementaire spécifique de l'artisanat (Assimakopoulou 1998). L'Italie s'est, quant à elle, industrialisée tardivement par rapport à la plupart des pays d'Europe occidentale, mais, contrairement à la Grande-Bretagne, l'industrialisation y a constitué un objectif national largement accepté, et les partis de droite comme de gauche se sont trouvés incités à soutenir les petites et moyennes entreprises industrielles (Trigilia 1986). 
Partant d'une situation de moindre développement, le taux de croissance de l'Italie depuis la fin de la guerre s'est montré le plus impressionnant des deux. En outre, si l'on prend comme mesure de la compétitivité des produits manufacturés le rapport exportations/importations, l'Italie a continuellement dépassé le RoyaumeUni. Ce dernier est plus performant dans le domaine des services marchands, mais pas assez pour compenser son retard dans les secteurs de l'agriculture et de la production industrielle. Ses exportations restent supérieures à celles de l'Italie en pourcentage du PIB, même en ce qui concerne les produits industriels, mais l'écart se resserre.

Si l'on examine de plus près les points forts des exportations, le Royaume-Uni obtient de meilleurs résultats dans les branches industrielles à base scientifique, tant celles à qualification élevée (comme la pharmacie, les instruments de précision, les équipements de télécommunication, l'industrie informatique) que l'industrie chimique de masse. L'Italie est en revanche plus performante pour les biens de consommation et notamment l'habillement et la chaussure. Elle marque également des points dans la fabrication de machines et de machines-outils. Elle dispose d'une solide industrie textile, un secteur à faible qualification où la grande majorité des pays avancés a du mal à se maintenir face aux bas prix des producteurs grecs, portugais ou du Tiers Monde, mais qui se trouve lié au secteur de l'habillement. Enfin, l'Italie surpasse la Grande-Bretagne dans un secteur industriel classique, celui de la production de masse de la grande entreprise : automobile, aéronautique et construction navale notamment.

Les deux pays ont toujours présenté des contrastes prononcés en matière de politique économique. Le Royaume-Uni est devenu, au lendemain de la Seconde Guerre mondiale, l'un des principaux adeptes de la politique keynésienne : gestion par la demande, compromis social et État-providence. L'Italie, par contre, avec sa forte opposition communiste exclue du pouvoir, n'a jamais totalement adhéré au consensus keynésien. Les deux pays ont connu des changements considérables d'orientation économique dans les années quatre-vingt et quatre-vingt-dix, mais dans des directions opposées, ce qui n'était pas de nature à réduire leurs différences. La droite néo-libérale britannique, arrivée au pouvoir après de grandes défaites politiques et syndicales de la gauche, s'est trouvée en mesure de réduire à néant l'influence politique du monde du travail et de désengager le pays du compromis social. Tandis que la fin de la guerre froide et le poids croissant des syndicats en Italie ont à la fois nécessité et permis la fin de l'exclusion politique de la gauche : on a pu commencer à aborder les réformes par le biais du compromis social. Mais l'Italie a dû, tout autant que la Grande-Bretagne, s'accommoder de l'orthodoxie néo-libérale dominante à l'échelle mondiale. Qu'en est-il donc aujourd'hui de ces deux pays à la fois comparables et si différents ? 


\section{Le Royaume-Uni et la conformité néo-libérale}

Dans les quatre premières décennies de l'après-guerre, la puissance des services financiers, point fort de la Grande-Bretagne, ne représentait pas un atout bien important sur le plan de la compétitivité et a même probablement nui à l'industrie. Les institutions financières entretenant avec l'élite politique des liens plus étroits qu'aucune branche industrielle ${ }^{1}$, la politique de la livre forte, appuyée par la finance, a habituellement prévalu, alors qu'elle allait souvent à l'encontre des préférences des entreprises industrielles (Longstreth 1987 ; Overbeek 1990 ; Talani 2000). Le consensus international des années quatre-vingt sur la libéralisation des marchés financiers, dopée par la capacité de réaction rapide que leur fournissaient les nouvelles technologies de l'information, est venu renforcer la domination de la City. Les économies ayant adopté l'approche nord-américaine des finances de l'entreprise détenaient désormais un avantage majeur. Ce modèle de concurrence loyale, valorisant l'actionnaire, s'adaptait mieux aux opérations internationales que les systèmes plus lourds de la plupart des autres pays (Story et Walter 1997). Les services financiers organisés sur cette base ont supplanté l'industrie pour devenir le secteur dominant des pays avancés. Cette évolution se produisait au moment même où la droite néolibérale britannique, s'appuyant sur les victoires qu'elle avait remportées sur la gauche politique et sur les syndicats, parvenait à se défaire du compromis social keynésien. Le désengagement vis-à-vis de l'intégration européenne et le rapprochement avec l'économie américaine sont venus couronner le tout.

L'effondrement de l'industrie qui en a été le corollaire a fait problème pendant la plus grande partie des années quatre-vingt (Hirst et Zeitlin 1988). Les néolibéraux parvenus au pouvoir à la fin des années soixante-dix avaient initialement prévu de relever l'industrie, mais ils ont dû infléchir leur politique devant la difficulté de la tâche, conjuguée aux nouvelles possibilités qu'offrait le secteur financier et au caractère très porteur de certains autres services (qui paraissaient exiger moins d'efforts d'infrastructure de la part de la puissance publique que l'industrie). Les programmes de soutien à l'industrie se sont pour la plupart limités à des exemptions d'impôt et à des mesures de stimulation des investissements japonais, coréens et américains. Les investisseurs britanniques préféraient les services.

Le Royaume-Uni cumule les meilleurs traits de deux systèmes très différents d'emploi tertiaire, l'américain et le scandinave (Esping-Andersen 1996 et 1999). L'américanisation des schémas de consommation (notamment la restauration rapide) a ouvert de nouvelles possibilités d'expansion de l'emploi, au surplus

\footnotetext{
1. La Banque d'Angleterre est, tant sur le plan institutionnel que spatial, au centre de la City. Elle entretient à son tour des rapports privilégiés avec le Trésor. Aucun autre intérêt économique organisé ne dispose d'une relation avec l'État britannique aussi solide et aussi formelle.
} 
favorisée par la traditionnelle division du travail selon les sexes en Grande-Bretagne. Les employeurs tirent parti du développement du travail à temps partiel, soutenu par la demande féminine dans ce domaine (Hakim 1998). La déréglementation du marché du travail a permis aux femmes de travailler le soir ou de diviser leur journée de travail. Par ailleurs, et malgré le néo-libéralisme ambiant, le système britannique de protection sociale et de services publics, un des piliers du compromis de l'après-guerre, s'est maintenu. Fait particulièrement important, il pratique davantage la prestation de services que les transferts de paiements, à l'instar des systèmes sociaux des pays scandinaves. Il est ainsi fortement créateur d'emplois, essentiellement féminins, dans l'enseignement, la santé et l'aide sociale. Cet emploi féminin est resté relativement inélastique, même lors de la période critique des années quatre-vingt qui a vu l'industrie britannique entrer dans une crise grave. La Grande-Bretagne est un des très rares pays au monde à avoir un taux de chômage féminin inférieur à celui des hommes.

Dans les années quatre-vingt-dix, le déclin de l'industrie et l'essor des services non marchands ont doté le Royaume-Uni d'une certaine capacité de résistance aux chocs économiques et aux pressions de la concurrence internationale, tout en se révélant porteurs en matière d'emplois. L'impossibilité du maintien de la livre dans le Système monétaire européen en 1991, initialement vécue comme une mise à l'épreuve de la solution néo-libérale, a paradoxalement permis à l'économie d'exploiter les avantages précédemment acquis. Émancipée du cycle économique européen, l'économie britannique a pu s'accrocher à celui des États-Unis, qui a suivi une pente de plus forte croissance pendant le reste de la décennie. Au début de l'an 2000 cependant, on pouvait craindre le retour des anciennes difficultés, du fait de tensions entre les orientations concurrentes de la politique monétaire, selon les intérêts de la City ou selon les besoins de l'industrie.

Le secteur industriel lui-même continue d'être dominé par de grandes entreprises (Crouch 1999, chap. 6) ${ }^{2}$. On a vu que la Grande-Bretagne a une position forte dans les secteurs industriels à base scientifique. L'économie britannique est souvent considérée - notamment par comparaison avec l'Allemagne - comme employant une main-d'œuvre peu qualifiée (Finegold et Soskice 1988 ; Prais 1981). Mais ce n'est pas vrai dans le cas des secteurs en question. La médiocrité des qualifications se situe dans les niveaux moyens, où ce sont les entreprises qui sont supposées les fournir. L'enseignement supérieur britannique produit depuis longtemps des cadres scientifiques et techniques peu nombreux, mais dotés d'une solide formation et très polyvalents (Crouch, Finegold et Sako 1999, chap. 2 et 4). Cet avantage - auquel sont venus s'ajouter l'impérieuse nécessité de changement consécutive à l'effondrement économique des années quatre-vingt et la souplesse induite par le système financier - a placé la Grande-Bretagne en position de tirer profit des secteurs consommateurs de connaissances, porteurs pendant les années quatre-vingt-dix : 
la biotechnologie, l'informatique, les industries culturelles (Swann, Prevezer et Stout 1998). L'emploi, et l'économie en général, semblent de plus en plus se partager entre des secteurs, petits mais solides, à forte qualification (dont le secteur financier et les professions du service public de santé, d'éducation et d'assistance), et les vastes domaines de services à faible qualification (travail de bureau non qualifié, commerce de détail, restauration rapide).

L'économie suit un schéma géographique de plus en plus prononcé (Crouch et Farrell 2000). L'industrialisation s'est initialement concentrée dans certaines régions, et il a toujours existé de fortes spécificités sectorielles régionales. Depuis le déclin des grandes industries du Nord au début du siècle, la richesse, l'innovation économique et la population se sont progressivement déplacées vers le Sud-Est. Les années quatre-vingt ont vu ce processus s'intensifier avec la poursuite de l'effondrement de l'industrie, l'essor des services financiers installés à Londres, et une évolution idéologique hostile à toute intervention sur les marchés. Le RoyaumeUni et la France ont cette caractéristique commune d'être dominés économiquement par leur capitale et sa région. La société post-industrielle, tendant à concentrer la croissance des services haut de gamme dans quelques centres métropolitains choisis, capables de devenir des cités à vocation mondiale (Scott 1998), renoue, en l'intensifiant, avec cet ancien processus temporairement interrompu par la révolution industrielle. Le sud-est de l'Angleterre (Grand Londres exclu) est la seule région du Royaume-Uni à avoir un revenu moyen par tête supérieur à la moyenne européenne. C'est aussi la région la plus riche de toute l'Union³.

On a longtemps considéré la prédominance du savoir-faire financier sur l'expertise technologique comme une faiblesse du gouvernement d'entreprise (corporate governance) en Grande-Bretagne, par comparaison avec l'Allemagne, le Japon ou la France. Or ce handicap s'est transformé en avantage dans un monde où les OPA hostiles et le poids de l'actionnaire sont devenus les facteurs tout-puissants de la conduite de l'entreprise. Le secteur public, puis certaines administrations de l'État ${ }^{4}$, ont été privatisés. La concurrence a été massivement introduite, mais n'empêche pas l'établissement d'oligopoles puissants résultant soit d'OPA hostiles soit de privatisations, délégations ou sous-traitances dans les domaines encore dominés par l'État. Le reste du service public a été remodelé à l'image des services marchands équivalents : ainsi, les hôpitaux et les médecins généralistes doivent entretenir entre

\footnotetext{
2. Il est difficile de faire un calcul précis car les statistiques britanniques ne prennent pas en compte les entreprises, mais les « établissements » (bâtiments ou même parties de bâtiment). Mais il semble clair que le Royaume-Uni a une proportion particulièrement faible de PME et particulièrement forte de groupes géants.

3. C'est à Londres qu'est produite la richesse, mais ce n'est pas là que vivent les riches... Le revenu moyen d'un Londonien se situe en dessous de la moyenne européenne.

4. Dans les secteurs où la privatisation des services est politiquement impossible, on en privatise parfois le contrôle, ce qui place le service public sous régulation privée. C'est le cas en particulier de l'éducation.
} 
eux des relations analogues à celles de la vente ou de l'achat de services.

La déréglementation du marché de l'emploi a été effective, grâce notamment à une nouvelle législation et à un affaiblissement considérable des syndicats dû au déclin de l'industrie et à une série de grands conflits, mais aussi à la logique même du système britannique de relations industrielles. Les syndicats britanniques ont longtemps privilégié la négociation collective par rapport à la législation, considérant qu'ils étaient en mesure d'obtenir plus par ce moyen, grâce à leurs propres forces. Lorsque leur puissance s'est trouvé ébranlée, le système s'est mis automatiquement à fonctionner à leur détriment. Ce qu'il restait de négociation collective s'est progressivement décentralisé au niveau de l'entreprise, plus sensible aux pressions du marché (Edwards et al. 1998). Le changement d'équilibre politique a conduit à l'abandon d'une fiscalité à objectifs égalitaires ; l'impôt est devenu plus défavorable aux petits revenus. À la fin des années quatre-vingt, le RoyaumeUni avait quitté la voie européenne et japonaise de réduction progressive des inégalités (avant et après impôt) pour emprunter celle, déjà bien établie aux ÉtatsUnis, du creusement des inégalités et de l'accentuation de la pauvreté (OCDE 1997). Le gouvernement travailliste de 1997 est revenu quelque peu sur cette tendance en instaurant un salaire minimum et une certaine reconnaissance des syndicats, ainsi qu'un modeste retour de l'État-providence. Mais l'orientation générale de la politique économique n'a pas changé.

Le Royaume-Uni est maintenant capable de produire des emplois à bas salaires dans les services de la distribution, les services personnels et la restauration rapide, emplois autrefois associés à des économies relativement «attardées » mais qui sont souvent, dans les années quatre-vingt-dix, la marque de pays parvenus à éluder les exigences d'une industrie forte. Ce réservoir d'emplois s'ajoute à celui que représente un système de protection sociale et de services publics centré sur les prestations plus que sur les transferts monétaires, ainsi qu'à celui d'un secteur de haute technologie en pleine expansion, pour donner à la Grande-Bretagne un avantage notable, en la matière, par rapport aux pays d'Europe continentale. En revanche, une grande partie des activités impliquant un haut niveau de technologie n'est encore qu'au stade de la pré-production. La Grande-Bretagne a depuis longtemps la réputation de laisser à des entreprises de pays étrangers l'exploitation commerciale d'innovations dues à l'excellence de ses formations universitaires.

\section{L'Italie et l'ambivalence néo-libérale}

À la fin des années soixante-dix, les petites entreprises d'Italie du Centre et du NordEst avaient tiré profit de la crise du fordisme (Bagnasco 1977 ; Becattini 1987 ; Trigilia 1986). Après avoir commencé à fonctionner avec du personnel relativement peu qualifié, de nombreux districts industriels se sont orientés vers le haut de 
gamme. Le système national d'enseignement y a moins contribué que des programmes de formation informels ou locaux. C'est ce secteur qui a développé l'ensemble de produits universellement connus sous l'étiquette made in Italy : habillement, chaussure, autres produits de la mode, meubles (Trigilia et Burroni 2000). La qualité du design et un souci d'adaptation constant pour rester à la pointe de la mode sont venus compenser la hausse des coûts dans la plupart de ces secteurs, la structure en petites entreprises s'étant révélée extrêmement flexible. Les besoins de ces branches sont par bien des aspects semblables à ceux des entreprises de haute technologie se développant aux États-Unis ou au Royaume-Uni, un secteur, toutefois, qui n'a que peu d'importance en Italie, exception faite de certains fabricants de logiciels. Les établissements publics d'enseignement scientifique et technique ne produisent pas une offre adéquate de personnel qualifié, et d'ailleurs ce type d'enseignement n'est pas à la portée des systèmes informels et locaux (Crouch, Finegold et Sako 1999, chap. 3 et 6). Le modèle italien de petites entreprises a cependant produit un profil de qualifications et une structure souple à l'écoute des clients qui conviennent parfaitement à la fabrication de machines. Au cours des années quatre-vingt, l'Italie était le seul pays occidental à résister au défi japonais dans le secteur des machines-outils (idem, chap. 3).

Le soutien de l'État aux infrastructures de cette économie dynamique est resté à la traîne. Les dépenses publiques de l'Italie ont pris de l'ampleur, mais selon un schéma très différent de celui de la Grande-Bretagne ou des pays scandinaves. Leur grande majorité était affectée à des transferts monétaires, souvent de type clientéliste, destinés à attacher certaines composantes de l'électorat à des partis politiques (Ferrera 1997). Le clientélisme vira à la corruption pure et simple et les Italiens, tenant généralement leur État en piètre estime, réagirent, d'une part (dans les districts industriels), en vivant leur vie économique et politique au niveau local, d'autre part, en s'adonnant massivement à l'évasion fiscale. Dans le Sud, beaucoup moins riche en entreprises et bien plus dépendant de l'État en ce qui concerne l'emploi, cette situation a favorisé le développement de la mafia et de ses connexions économiques et politiques.

Gaspillages dans la dépense publique, recettes fiscales inappropriées, État peu performant mais très présent ont eu pour conséquences une faiblesse des infrastructures et un endettement public chronique qui ont fini par affecter la vitalité de l'industrie. Ce seront des chocs externes qui susciteront des changements. L'un de ces chocs, partagé avec le Royaume-Uni, quoique avec des conséquences très différentes, fut la sortie de la monnaie du Système monétaire européen en 1991 ; l'autre, l'effondrement de l'Union soviétique en 1989.

Le Parti communiste italien avait pris ses distances vis-à-vis de l'URSS dès les années soixante-dix, ce qui faisait déjà de lui un partenaire plus acceptable pour les autres partis. L'effondrement final du système soviétique a parachevé son évolution, 
tout en rendant désormais superflu, tant sur le plan interne qu'externe, de protéger la classe politique en général. Brusquement, les magistrats italiens eurent accès à des renseignements sur la mafia et la corruption des hommes politiques. Ce fut le prélude à une campagne se soldant par des arrestations, des procès criminels, et une grave crise d'organisation et de leadership tant dans la mafia que dans l'élite politique.

Alors que la Grande-Bretagne réagissait à la crise financière de 1991 en se rapprochant des États-Unis et en s'éloignant de l'UE, l'Italie ressentit comme un enjeu national majeur le retour « au cœur de l'Europe ». Un consensus presque général se dégagea au sein des élites économiques et politiques, des syndicats et de l'opinion sur le fait que le pays se devait d'être à l'avant-garde de l'Union monétaire européenne (Talani 2000). À la lutte contre la corruption politique succéda une importante restructuration des finances publiques pour réduire le poids du déficit des comptes publics et de l'État, et pouvoir ainsi rejoindre l'Union monétaire. Les gouvernements - désormais dominés par les héritiers du Parti communiste purent ainsi exiger de la population des sacrifices budgétaires et sociaux considérables au nom de l'Europe.

Les conséquences de cette politique s'avérèrent aussi paradoxales, bien que de façon opposée, qu'au Royaume-Uni. Comme dans la plupart des autres pays candidats à l'UEM, la pression fiscale eut un effet négatif sur la demande intérieure et donc sur la croissance des services non marchands, grande source d'emplois des économies avancées aujourd'hui. Parallèlement, l'État n'était pas en mesure d'augmenter le nombre des emplois publics et le chômage resta très élevé, notamment celui des femmes dans le sud du pays. Mais l'inflation, et donc les taux d'intérêt, demeurèrent modestes. Les entreprises industrielles, petites et grandes, commencèrent à se porter mieux. Lorsque l'euro fut enfin lancé, sa chute sur les marchés des changes profita largement aux exportateurs italiens. La balance des paiements poursuivit son redressement, la santé de l'industrie se confirma mais au prix de réductions d'emplois, corollaire contemporain de la compétitivité. L'Italie se trouva pourvue d'une industrie florissante, d'un secteur tertiaire faible et d'un chômage élevé, à l'exact opposé de la situation britannique.

Dans l'intervalle, les responsables politiques et économiques italiens ont adhéré à la même orthodoxie mondiale de déréglementation et de priorité au secteur financier que les britanniques, sans être néanmoins, comme ces derniers, à la pointe de changements apparemment peu conformes aux structures fondamentales de leur économie. Les grandes entreprises italiennes avaient prospéré grâce à la protection politique dont elles bénéficiaient, et les petites, grâce à leur capital familial et aux réseaux de relations personnelles existant entre les banques et les entreprises industrielles. Le passage au modèle américain de prééminence de l'actionnaire pose de graves problèmes aux premières comme aux secondes. Du fait de ces 
pressions et des difficultés croissantes rencontrées par les petites entreprises sur les marchés globalisés, le modèle fondé sur la petite entreprise se rapproche du schéma japonais, avec une grande entreprise coordonnant un ensemble de petits fournisseurs (Trigilia et Burroni 2000).

D'autres éléments du programme anglo-américain sont également repris. L'Italie privatise des entreprises du secteur public. Celles-ci sont souvent vendues à des groupes bénéficiant de relations personnelles au gouvernement, et sans réel démantèlement des actifs, mais cela est également vrai d'un certain nombre de privatisations en Grande-Bretagne. Une politique de concurrence destinée à empêcher les collusions a été mise en place et dotée de puissants organes d'application. Elle a eu le mérite considérable d'attirer l'attention de la population sur les tendances à la cartellisation d'une grande partie de l'économie italienne mais n'a obtenu que des résultats pratiques mitigés, les professions libérales opposant de fortes résistances. Les grandes entreprises publiques et privées semblent rester à la traîne du reste de l'Europe en matière d'écoute des besoins de leurs clients. En revanche, des travaux récents des organismes de concurrence britanniques indiquent que les prix d'un certain nombre de biens faisant l'objet d'échanges internationaux, notamment les véhicules, tendent à être plus élevés en Grande-Bretagne que dans la grande majorité des pays d'Europe occidentale, y compris l'Italie (Competition Commission 2000). La relation entre les pratiques des entreprises selon leur nature privée ou publique et la projection d'une image de sensibilité aux besoins des clients, d'une part, et la réalité, de l'autre, ne laisse pas d'être complexe.

Dans les services publics italiens, l'alignement sur des modèles de marché et les réformes en général sont très peu avancés. Les fonctionnaires constituent un groupe privilégié. Les partis au pouvoir et un syndicalisme vigoureux ont renforcé le modèle du XIX ${ }^{\mathrm{e}}$ siècle, où le service de l'État revêt un caractère exceptionnel, les partis utilisant l'emploi public comme moyen d'attirer et de récompenser des sympathisants et les syndicats de fonctionnaires s'organisant souvent de façon catégorielle, en dehors des principales centrales. C'est aussi la fréquence des situations d'impasse politique qui a empêché les réformes. Le compromis conduit souvent à l'établissement de nouvelles structures à côté des anciennes, avec pour conséquence une prolifération d'organismes parallèles. Le nombre d'employés, le coût des services et la complexité administrative s'en trouvent multipliés, mais non, tant s'en faut, l'efficacité.

La déréglementation du marché du travail et l'affaiblissement de l'État-providence soulèvent également des difficultés. Après des décennies d'exclusion politique des syndicats et de violents conflits sociaux, l'Italie a mis au point l'un des modèles de partenariat social les plus performants d'Europe (Regini et Regalia 1996). Les dirigeants syndicaux, le gouvernement et une grande partie du patronat adhèrent aux principaux objectifs politiques en matière d'intégration à l'Union monétaire, 
de réforme du système de retraites et de modernisation de l'économie, bien que leur application pose problème à des niveaux moins élevés. Les coalitions gouvernementales et les patrons sont pour la plupart réticents à détruire les acquis. La modification de la réglementation du marché du travail doit donc s'effectuer à petits pas et procéder d'un accord généralisé, ce qui vient retarder les progrès sur des points comme l'emploi féminin ou le travail à temps partiel.

La négociation collective s'est décentralisée, ce qui répond à la préférence de l'économie italienne pour le local et convient aux militants syndicaux et aux dirigeants d'entreprise. Cette évolution va toutefois à l'encontre de la concertation qui a permis des pactes sociaux, une réforme du système de protection sociale et une évolution modeste du marché de l'emploi.

À un autre niveau, l'association d'une réglementation stricte et tatillonne en matière d'emploi et de son contournement massif aboutit à une déréglementation de fait qui masque le fonctionnement réel du marché de l'emploi. Un grand nombre de personnes ayant pris une retraite très largement anticipée grâce au système exorbitant de pensions qui a temporairement prévalu pendant les années quatre-vingt continuent de travailler, mais au noir, sous peine de perdre leurs indemnités. Beaucoup d'autres activités s'effectuent à l'insu du fisc, et se trouvent ainsi également exclues de la réglementation du marché du travail. Ni le niveau réel de réglementation, ni celui du chômage, ne sont donc reflétés par les indicateurs officiels, surtout dans le Sud. Là encore, l'existence de solutions informelles commodes s'allie aux difficultés du processus de réforme pour empêcher la réalisation de changements formels.

La répartition de la richesse en Italie est mal connue. Elle est probablement moins inégale qu'au Royaume-Uni. La classe des petits capitalistes y est bien plus nombreuse, ce qui semble indiquer une fragmentation de la propriété du capital. Quant aux revenus, ils sont très inégalement distribués (Crouch 1999, chap. 6 et 13). Les disparités régionales et les inégalités extrêmes en matière d'enseignement expliquent probablement l'écart des revenus bruts. Par ailleurs, le système de protection sociale, budgétisé ou non, n'a guère d'effet sur la répartition nette dans la mesure où il affecte l'essentiel de ses dépenses aux retraites des catégories moyennes et à l'entretien du clientélisme. L'Italie présente donc depuis longtemps un profil de répartition des revenus avant et après l'intervention de l'État plus proche de celui des États-Unis que la grande majorité des pays européens (Crouch 1999, chap. 13). Elle a, de ce fait, moins de chemin à faire pour se conformer au nouveau modèle américain. D'un autre côté, avec l'amélioration des niveaux d'éducation et l'avènement du partenariat social pendant les années soixante-dix et quatre-vingt, l'Italie avait commencé à devenir plus égalitaire. Elle s'est mise plus tard que le Royaume-Uni à inverser les tendances égalitaristes de la période keynésienne, et procède dans cette direction plus lentement qu'il ne l'a fait. 


\section{Des paradoxes qui perdurent}

Les voies différentes empruntées à l'heure actuelle par l'Italie et le Royaume-Uni révèlent un découplage entre la compétitivité internationale et la croissance de l'emploi dans une économie post-keynésienne et post-industrielle. Il existe un second paradoxe. Bien qu'on considère la Grande-Bretagne comme plus proche du paradigme libéral, l'Italie est en fait plus conforme au modèle d'économie industrielle à base de petites entreprises, proche du marché, flexible et capable d'initiatives qu'évoque ce paradigme. L'explication la plus plausible qu'on puisse avancer est que le rôle dynamique des petites entreprises, au moins dans l'industrie, exige divers types de soutien institutionnel et non le seul jeu du marché (Bagnasco 1988 ; Trigilia 1986, 1999). Le modèle italien fondé sur les petites entreprises présente l'avantage de bénéficier principalement d'appuis informels et donc ignorés par la stratégie de déréglementation, qui se concentre sur l'action de l'État. Cette situation changerait néanmoins si certaines structures de base venaient à disparaître, comme les codes de l'artisanat. Ce modèle a été ébranlé ces dernières années par l'émergence de grandes entreprises organisées en réseaux, phénomène qui n'est pas assez marqué pour menacer la concurrence mais qui est révélateur de l'ambiguité des relations entre la théorie de la liberté des marchés, laquelle suppose un grand nombre de producteurs anonymes, et la réalité de la pression des marchés, qui tend à réduire le nombre de ces producteurs.

Il est vrai, toutefois, que le schéma italien de petites entreprises et le modèle de grandes firmes associé au capitalisme britannique remettent en cause certains principes fondamentaux des théories néo-classique et néo-libérale, notamment leur insistance sur l'anonymat et le fait qu'elles privilégient les relations résultant du seul marché plutôt que les relations personnelles. Remarquons tout d'abord que, si elle ne s'occupe pas de fabrication, la City de Londres possède presque toutes les autres caractéristiques d'un district industriel italien, et que ses relations avec les centres du pouvoir politique jouent toujours un rôle essentiel dans la formation d'un environnement à sa convenance. Ensuite, les très grandes entreprises mettent plus que largement à profit la capacité que leur donne leur taille d'agir en dehors des strictes contraintes du marché, mais naturellement à un niveau différent de celui des réseaux de réciprocité des petites entreprises des districts industriels. À titre d'exemple, les privatisations et l'évolution dans le sens du marché ont permis à des entrepreneurs, tant en Italie qu'en Grande-Bretagne, de jouer de leur influence auprès des pouvoirs publics pour obtenir des contrats. Il s'agit d'un des paradoxes du néo-libéralisme : malgré ses fortes affinités avec le néo-classicisme, il suppose la victoire politique d'intérêts pro-capitalistes qui ne seront pas nécessairement très enclins par la suite à se soumettre eux-mêmes à la discipline de l'économie néoclassique.

Traduit de l'anglais par Marie-Pierre Émery 


\section{Bibliographie}

Assimakopoulou (Z.), 1998. The Development of Small Business Policy in Britain 1970-1990, thèse, Florence, Institut universitaire européen.

Bagnasco (A.), 1977. Tre Italie, Bologne, II Mulino.

BAgnasco (A.), 1988. La costruzione sociale del mercato, Bologne, Il Mulino.

BeCATtINI (G.) (dir.), 1987. Mercato e forze locali : il distretto industriale, Bologne, II Mulino.

Competition Commission, 2000. Prices of Motor Vehicles, Londres, HMSO.

CROUCH (C.), 1999. Social Change in Western Europe, Oxford, Oxford University Press.

Crouch (C.), Farrell (H.), 2000. "Great Britain : Falling through the holes in the network concept ", dans C. CROuCH et al. 2000.

Crouch (C.), Finegold (D.), SAKo (M.), 1999. Are Skills the Answer ? The Political Economy of Skill Creation in Advanced Industrial Countries, Oxford, Oxford University Press.

CRouch (C.), Le Galès (P.), Trigilia (C.), Voelzkow (H.), 2000. Local Production Systems in Europe : Rise or Demise?, Oxford, Oxford University Press.

Edwards (P.), Hall (M.), Hyman (R.), Marginson (P.), Sisson (K.), Waddington (J.), Winchester (D.), 1998. " Great Britain : From partial collectivism to neo-liberalism to where ? ", dans A. FerneR, R. HyMAn (eds.), Changing Industrial Relations in Europe, Oxford, Blackwell, pp. 1-54.

EsPInG-ANdersen (G.) (ed.), 1996. Welfare States in Transition : National Adaptations in Global Economies, Londres, Sage.

Esping-Andersen (G.), 1999. Social Foundations of Post-Industrial Economies, Oxford, Oxford University Press.

Ferrera (M.), 1997. Le trappole del welfare, Bologne, II Mulino.

FineGold (D.), SOSKICE (D.), 1988. « The failure of training in Britain : Analysis and prescription », Oxford Review of Economic Policy 4 (3), pp. 21-53.

HAKIM (K.), 1998. Social Change and Innovation in the Labour Market, Oxford, Oxford University Press. HIRST (P.), ZeItLIN (J.), 1988. Reversing Industrial Decline, Oxford, Berg.

LONGSTRETH (F.), 1987. The City, Industry, and the State, thèse, Université de Londres.

OCDE, 1997. Perspectives d'emploi, Paris, OCDE.

OVerbeEk (H.), 1990. Global Capitalism and National Decline: The Thatcher Decade in Perspective, Londres, Unwin Hyman.

Prals (S.J.), 1981. "Vocational qualifications of the labour force in Britain and Germany ", National Institute Economic Review, 98.

REGINI (M.), RegALIA (I.), 1996. Italia anni '90 : Rinasce la concertazione, Milan, IRES.

ScotT (A.), 1998. Regions and the World Economy, Oxford, Oxford University Press.

StORY (J.), WALTER (I.), 1997. Political Economy of Financial Integration in Europe: The Battle of the Systems, Manchester, Manchester University Press.

SWANn (G.M.), Prevezer (M.), Stout (D.), 1998. The Dynamics of Industrial Clustering : International Comparisons in Computing and Biotechnology, Oxford, Oxford University Press.

TALANI (L.S.), 2000. Betting for and against EMU : Who Wins and Who Loses in Italy and in the UK from the Process of European Monetary Union, Aldershot, Ashgate.

TRIGILIA (C.), 1986. "Small-firm development and political sub-cultures in Italy ", European Sociological Review, 2/3, pp. 161-175.

TRIGILIA (C.), 1999. "Capitale sociale e sviluppo locale », Stato e Mercato, 3, pp. 419-440.

TRIGILIA (C.), BURRONI (L.), 2000. "Local production systems in the economic development of Italy ", dans C. Crouch et al. 2000

WIENER (M.J.), 1981. English Culture and the Decline of the Industrial Spirit 1850-1980, Cambridge, Cambridge University Press. 